\title{
Determination of backscattered neutrons/gammas from open beam port of a research reactor
}

\author{
$1^{\text {st }}$ Anže Jazbec \\ Reactor Infrastructure Centre \\ Jožef Stefan Institute \\ Ljubljana, Slovenia \\ anze.jazbec@ijs.si \\ $4^{\text {th }}$ Klemen Ambrožič \\ Reactor Physics Department \\ Jožef Stefan Institute \\ Ljubljana, Slovenia \\ klemen.ambrozic@ijs.si
}

\author{
$2^{\text {nd }}$ Bor Kos \\ Reactor Physics Department \\ Jožef Stefan Institute \\ Ljubljana, Slovenia \\ bor.kos@ijs.si \\ $5^{\text {th }}$ Luka Snoj \\ Reactor Physics Department \\ Jožef Stefan Institute \\ Ljubljana, Slovenia \\ luka.snoj@ijs.si
}

\author{
$3^{\text {rd }}$ Vladimir Radulović \\ Reactor Physics Department \\ Jožef Stefan Institute \\ Ljubljana, Slovenia \\ vladimir.radulovic@ijs.si
}

\begin{abstract}
Neutron and gamma dose rate calculations were carried out around horizontal beam tube no. 5 at the Jožef Stefan Institute (JSI) TRIGA Mark II research reactor. Results were compared to the experimental measurements in order to verify the computation model. In addition, another set of calculations and measurements was carried out, where an additional shield made out of concrete and paraffin was installed. With that configuration, we were able to study neutron and gamma scattering.
\end{abstract}

Keywords-Reactor Physics, MCNP, ADVANTG

\section{INTRODUCTION}

In this article, a major step towards calculating dose rates using the Monte Carlo particle transport code MCNP [1] in reactor hall of JSI TRIGA reactor is presented. In recent years, Monte Carlo simulations were used to calculate various parameters in or around JSI TRIGA core [2][3]. The computational model was experimentally verified using several experiments to prove the credibility of the calculations [4]. However, far from the core Mote Carlo simulations give statistically insignificant results in a reasonable simulation time. In order to shorten calculation time, variance reduction techniques were used. Dose rates in the reactor hall around beam tube no. 5 were calculated for the first time. This was achieved by accelerating MCNP calculations with ADVANTG code [5].

\section{MCNP}

Stochastic neutron transport calculations were performed with the MCNP code. Monte Carlo N-Particle (MCNP) is a program package used to simulate transport of neutrons, photons (gamma rays), electrons etc. In this case, it was used to simulate neutrons and prompt gamma rays emitted from an operating reactor. The Monte Carlo method is used to simulate a great number of particles in a desired computer model describing the geometry of the problem including material composition. The final result is an average value of a large number of events of interest in selected regions of the model. The main advantage of MCNP over deterministic methods is that geometry can be defined accurately and that MCNP handles energy and angular distribution of particles continuously. One of the drawback is long calculation times, therefore powerful computers are needed.
For the ENDF/B-VII.1 nuclear data library [6] was used in our calculations. All nuclear data is at room temperature as reactor was operated at low power).

\section{ADVANTG}

In this problem, we were interested in a region that is far from the particle source (reactor core) and expected dose rate values are about 7 orders of magnitude lower than in the core. Such problems can not be tackled with analog MC transport.

By analog ${ }^{1}$ we mean a simulation without any variance reduction techniques. Using analog MCNP calculation There was a need to introduce variance reduction parameters. One of the options is to use a method, where a population of particles is manipulated using so called weight windows. The weight of the particles is manipulated according to its energy and location. In ADVANTG the user defines a 3D Cartesian mesh over the MCNP model. Where materials are inhomogeneous, a finer mesh is needed and the same goes for the region close to the interested tallies. The idea is to have voxels as homogenous by material as possible in regions that are important for particle transport since each voxel will be represented by single weight. In addition, user has to define also parameters like library and Denovo [7] parameters. The code then uses deterministic transport code Denovo to determine forward and adjoint fluxes that are used to generate weight windows and source biasing. For each cell or voxel, an upper and lower bound are specified. If the particle, that enters the cell has weight higher than upper bound, it is split in a way that new particles weights are within the bounds. If the particle weight is below the lower bound, Russian roulette is used to either increase the particle weight or to kill it.

To achieve dose rate calculation with uniformly low statistical uncertainty in a Monte Carlo simulation, the distribution of Monte Carlo particles should be uniform throughout the interested region. Although this is not a "physical" response, it does intuitively represent a desirable objective for obtaining uniform uncertainty. In the ForwardWeighted Consistent Adjoint Driven Importance Sampling (FW-CADIS [8]) method, forward information (e.g., flux, dose) is used to define an appropriate response (adjoint source) to be used in a deterministic adjoint calculation. The weighted adjoint source is used to generate the adjoint importance function for achieving uniform particle density (or

\section{${ }^{1}$ A simulation without any variance reduction techniques.}

(C) The Authors, published by EDP Sciences. This is an open access article distributed under the terms of the Creative Commons Attribution License 4.0 (http://creativecommons.org/licenses/by/4.0/). 
response) throughout the system. With this method, it is possible to accelerate neutron transport calculations throughout the geometry of the problem. In addition, the method is applicable to calculation of multiple individual responses, depending on the adjoint source definition.

\section{COMPUTER MODEL AND MCNP INPUT}

Previous TRIGA reactor models [2] - [4] were focused on modelling the core and ex-core irradiation facility well inside the biological shield. In order to model radiation outside the biological shield a completely new computational model of the JSI TRIGA Mark II reactor was developed. Original blue prints of the reactor body, reactor components and reactor hall were taken as a basis. However due to several modifications through the years of operation, modified component dimensions were verified by actual measurements. Gathered data were used to develop a detailed CAD (Computer assisted design) model of JSI TRIGA reactor [9]. The CAD model consist of fuel elements, graphite reflector, irradiation channels, control rods, nuclear instrumentation, reactor pool, reactor body, beam tubes, thermal column, thermalizing column, dry chamber, additional concrete shield in reactor hall, complete reactor basement, reactor platform, spent fuel pool and reactor building (Fig. 1).

The CAD model was then transformed to a MCNP model cell by cell using routines written in Grasshopper [10]. Only
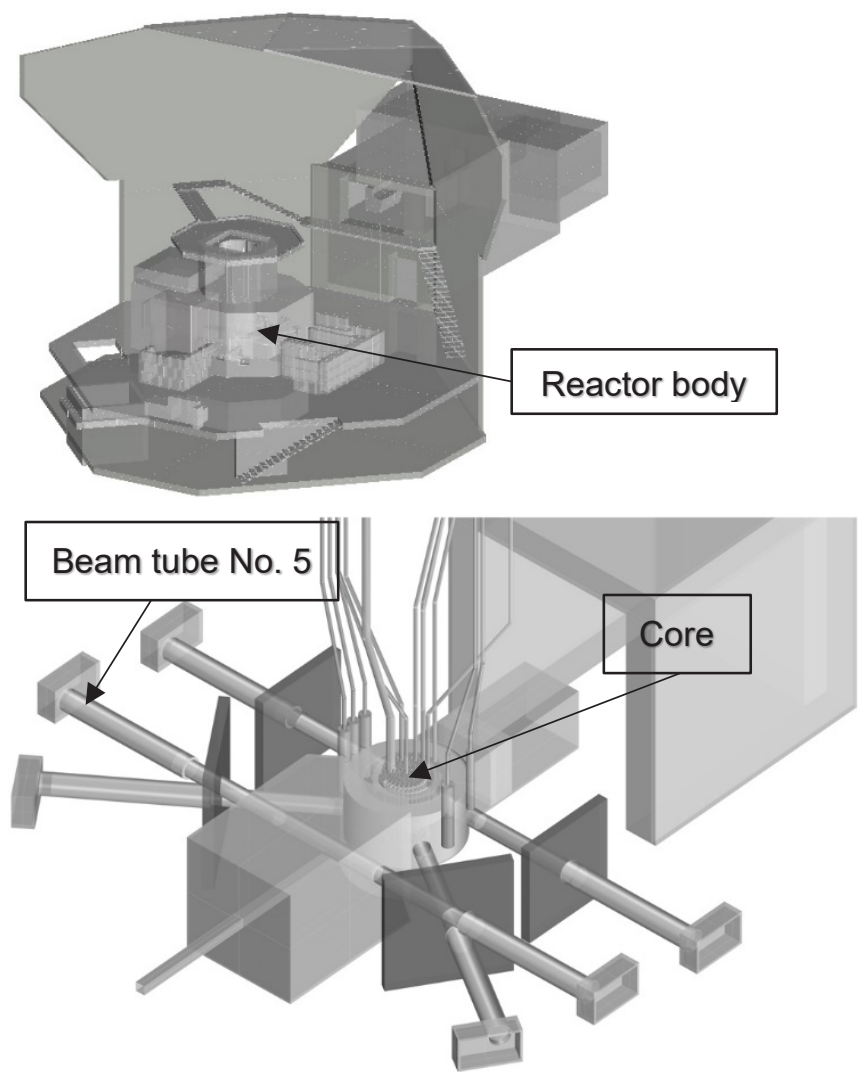

Fig. 1. CAD model of JSI TRIGA Mark II reactor. Up: some walls were removed for clearer presentation. Bottom: detailed modelling inside reactor body.

minor simplifications were done that we believe do not affect final results (e.g. stairs and control room are missing in MCNP model).

Since ADVANTG cannot be run in an eigenvalue calculation mode, a detailed neutron source had to be defined.
For that reason, an MCNP eigenvalue calculation was first performed to determine the neutron source. Each fuel element was divided into 100 discs and for each disc neutron fission rates were calculated. Obtained distributions were then used for neutron source definition. In this case, no gamma source was defined since only prompt gamma particles were simulated. In order to verify the newly calculated source, a neutron flux across the core was calculated and compared to the one gained by a criticality calculation (Fig. 2). Relatively short run was done, only $10^{8}$ particles were simulated in both cases, however good agreement can be observed. Differences are usually within 2 percent, mostly due to the statistical error. This calculation served as a quick and efficient verification of the source.

\section{EXPERIMENT:}

In order to validate the computational model and calculated dose rates and experiment was performed in which we unplug beam tube no. 5 and measured neutron and gamma dose rates in the beam. Beam tube no. 5 was selected because it is not facing the core directly, therefore there is low gamma background during reactor shutdown (Fig. 3). The beam port is located tangentially to the reactor core and pierces the thermal column, a large graphite block which is partly inside reactor poll, partly inside reactor concrete body. During normal reactor operation, the beam tube is plugged by two plugs that are made from concrete and wood respectively.

To study back-scattering of particles inside reactor hall, additional shield was installed $2.4 \mathrm{~m}$ away from the beam tube entry. Shield consisted of $30 \mathrm{~cm}$ of paraffin and $30 \mathrm{~cm}$ of concrete. In order to observe the effect of the shield,

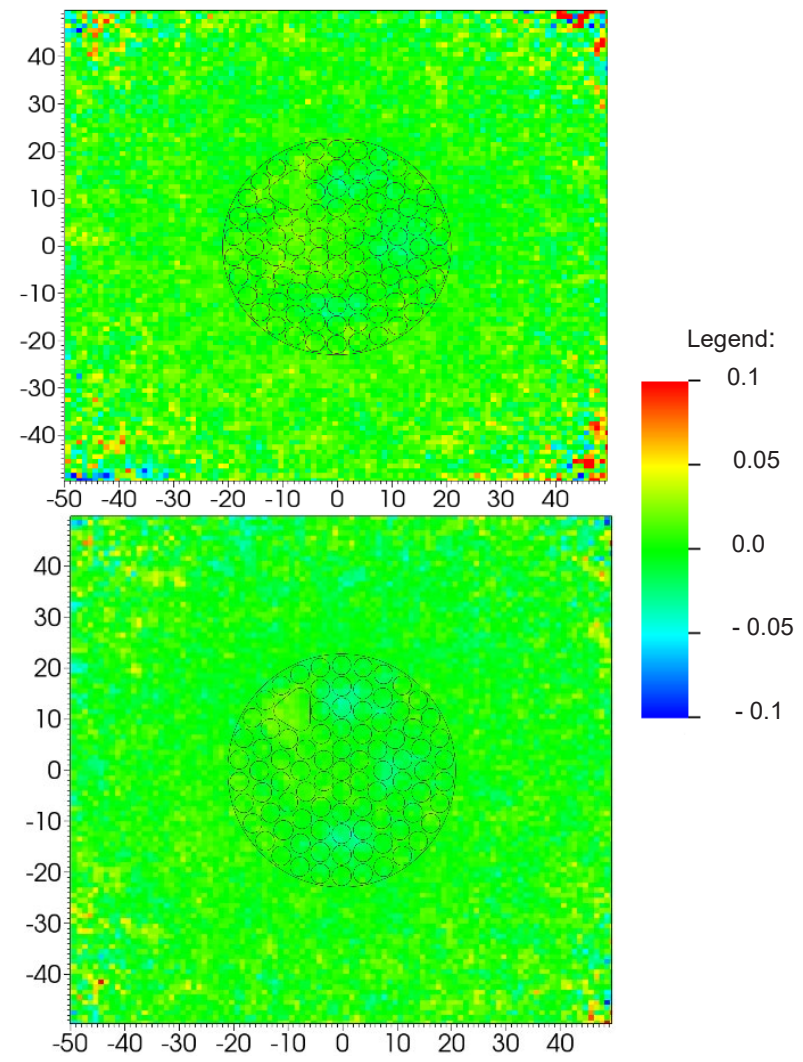

Fig. 2. Total neutron flux across reactor core was calculated for two different sources, KCode and SDEF, and compared. Relative difference of the KCode and SDEF sources is presented at the bottom (bottom) and top (top) part of the core. 


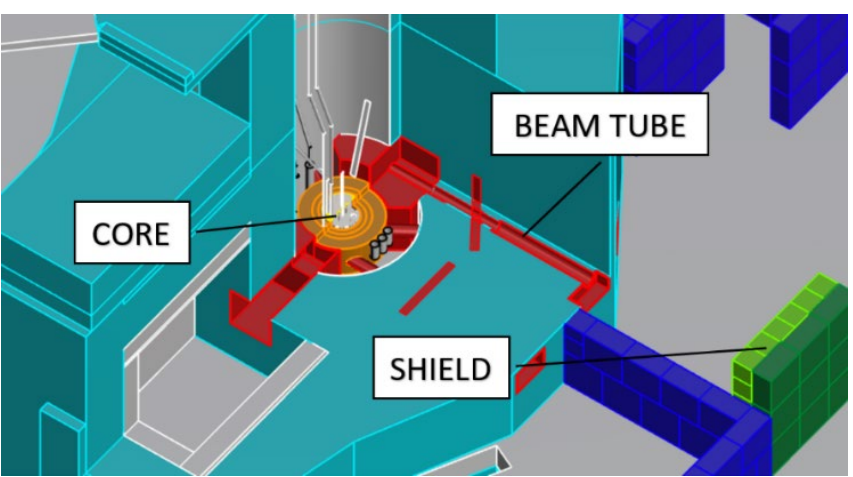

Fig. 3. Geometry of the beam tube location. With green colour the removable shield is noted. One can see tangential beam tube, that is piercing thermal column and dose not pierce reactor core.

measurements were compared to the one taken when there was no shield.

Neutron and gamma dose rates were measured at the locational presented in Fig. 4 and Table 1. Gamma and neutron dose rates were measured using Automess 6150AD probe and Berthold LB 123 Handheld Neutron Monitor respectively. Both detectors are regularly calibrated to measure $\mathrm{H}^{*}(10)$ and are part of accredited laboratory. For each location, three consecutive measurements were taken.

The first set of measurements was taken during reactor shutdown in order to determine background levels. Gamma levels were close to natural background; neutrons could not be detected due to shutdown of the reactor. The second set of measurements was taken during reactor operation at $500 \mathrm{~W}$ with the shield installed. For the last set of measurements, the shield was removed and reactor was once again operated at $500 \mathrm{~W}$. All results are presented in Table 1. Measuring error was determined in the following way: In case of measuring neutron dose, the result is given as counts per second (cps). Therefore, error is estimated as square root of numbered counts. In case of gamma dose rate measurement, error is estimated to $20 \%$ in case the dose rate is higher than $1 \mu \mathrm{Sv} / \mathrm{h}$. If the dose rate is lower, the error is estimated to $25 \%$. That is compliant with valid procedures used by our accredited laboratory.

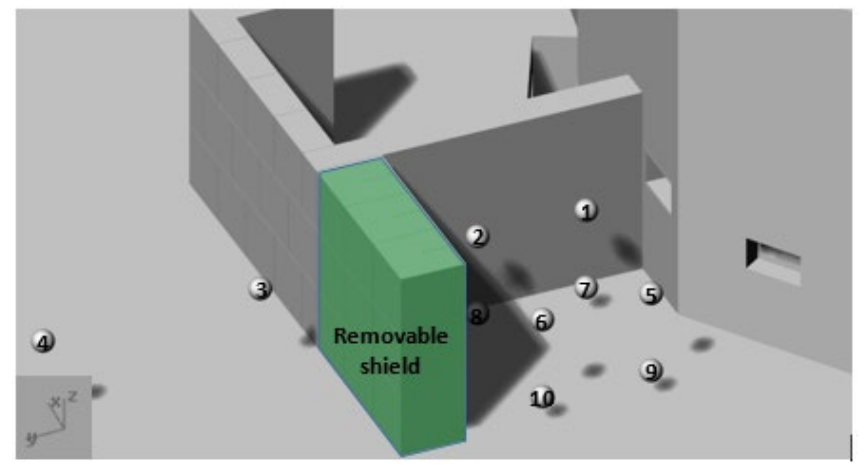

Fig. 4. Gamma and neutron dose rates were measured at 10 locations, displayed by spheres in the figures above.

\section{Vi. CAlculation}

For calculations, MCNP accelerated by ADVANTG was used. ADVANTG was used for variance reduction. The whole model was meshed and for each voxel, ADVANTG atomically generated weight-windows that are proportional to the adjoint flux in relevance with interested region. In the TRIGA MCNP model spheres were modelled according to Fig. 4. Neutron and gamma flux was calculated in these spheres. For gamma dose rates, ICRP conversion factors [10] were already included in the input file, so the results were proportional to the dose rate in $\mathrm{Sv} / \mathrm{h}$. To get a direct comparison with experimental results, MCNP results had to be re-normalized to the reactor power by multiplying them by factor S [11]:

$$
S=\frac{P \bar{v}}{w_{f} k}
$$

Where $\mathrm{P}$ represents reactor power, $\bar{v}$ is the average number of neutrons released by fission and equals $2.44, w_{f}$ is the average energy released during fission and is about $200 \mathrm{MeV}$ and $\mathrm{k}$ stands for the multiplication factor.

The neutron detector was set to measure fast neutrons response factor for Cf-251 was used. Since our spectra was not mono-energetic, measured dose rates were divided by a factor for fast neutrons (1.27 [14]) so we obtained result in counts per second (cps). Calculated spectra was multiplied by appropriate response factors that can be found in the manual [14]. Therefore, obtained numbers are once again cps so they can be directly compared to measured results. All calculated results are presented in Table 1. Variance given in Table 1 is statistical only.

TABLE I. MEASURED AND CALCULATED DETECTOR RESPONSES

\begin{tabular}{|c|c|c|c|c|c|c|c|c|c|c|c|c|c|c|c|c|c|}
\hline \multirow[b]{3}{*}{ No. } & \multirow[b]{3}{*}{$\mathrm{X}, \mathrm{Y}, \mathrm{Z}[\mathrm{cm}]$} & \multicolumn{8}{|c|}{ Measurements } & \multicolumn{8}{|c|}{ Calculations } \\
\hline & & \multicolumn{4}{|c|}{ Shield installed } & \multicolumn{4}{|c|}{ Shield removed } & \multicolumn{4}{|c|}{ Shield installed } & \multicolumn{4}{|c|}{ Shield removed } \\
\hline & & $\begin{array}{c}v \\
{[\mu \mathrm{Sv} / \mathrm{h}]}\end{array}$ & $\begin{array}{l}\text { Uncert. } \\
{[\mu \mathrm{Sv} / \mathrm{h}]}\end{array}$ & $\begin{array}{c}\text { Neutron } \\
\text { [cps] }\end{array}$ & $\begin{array}{c}\text { Uncert. } \\
\text { [cps] }\end{array}$ & $\begin{array}{c}\gamma \\
{[\mu S v / h} \\
]\end{array}$ & $\begin{array}{l}\text { Uncert. } \\
{[\mu \mathrm{Sv} / \mathrm{h}]}\end{array}$ & $\begin{array}{c}\text { Neutron } \\
\text { [cps] }\end{array}$ & $\begin{array}{c}\text { Uncert. } \\
\text { [cps] }\end{array}$ & $\begin{array}{c}v \\
{[\mu \mathrm{S} v / h]}\end{array}$ & $\begin{array}{c}\text { Varian. } \\
{[\%]}\end{array}$ & $\begin{array}{c}\text { Neutron } \\
\text { [cps] }\end{array}$ & $\begin{array}{c}\text { Varian. } \\
\text { [\%] }\end{array}$ & $\begin{array}{c}v \\
{[\mu \mathrm{Sv} / \mathrm{h}]}\end{array}$ & $\begin{array}{c}\text { Varian. } \\
{[\%]}\end{array}$ & $\begin{array}{c}\text { Neutron } \\
\text { [cps] }\end{array}$ & $\begin{array}{c}\text { Varian. } \\
\text { [\%] }\end{array}$ \\
\hline 1 & $0,400,108.6$ & 147 & 29.4 & 86.6 & 4.8 & 145 & 28.9 & 93.7 & 4.96 & 118 & 0.46 & 86.8 & 0.45 & 118 & 0.48 & 86.7 & 0.46 \\
\hline 2 & $0,500,108.6$ & 66.6 & 13.3 & 55.4 & 3.8 & 68.34 & 13.7 & 60.9 & 4.00 & 65.9 & 0.47 & 44.6 & 0.45 & 65.3 & 0.49 & 44.3 & 0.46 \\
\hline 3 & $0,700,108.6$ & 0.99 & 0.25 & 0.00 & I & 21.96 & 4.39 & 21.26 & 2.36 & 0.75 & 0.81 & 0.003 & 1.12 & 28.0 & 0.5 & 16.6 & 0.47 \\
\hline 4 & $0,900,108.6$ & 0.48 & 0.12 & 0.00 & I & 11.95 & 2.39 & 10.00 & 1.62 & 0.29 & 0.56 & 0.004 & 1.38 & 15.8 & 0.51 & 8.35 & 0.47 \\
\hline 5 & $-138,400,108.6$ & 1.03 & 0.21 & 0.37 & 0.31 & 0.36 & 0.09 & 0.10 & 0.16 & 0.19 & 2.2 & 0.09 & 0.56 & 0.14 & 0.99 & 0.08 & 0.6 \\
\hline 6 & $-138,500,108.6$ & 0.81 & 0.20 & 0.42 & 0.33 & 0.48 & 0.12 & 0.31 & 0.29 & 0.26 & 0.61 & 0.12 & 0.56 & 0.17 & 0.64 & 0.10 & 0.58 \\
\hline 7 & $0,400,30$ & 1.79 & 0.36 & 1.20 & 0.56 & 0.59 & 0.15 & 0.31 & 0.29 & 0.51 & 0.58 & 0.26 & 0.55 & 0.39 & 0.68 & 0.22 & 0.58 \\
\hline 8 & $0,500,30$ & 2.47 & 0.49 & 2.05 & 0.73 & 0.77 & 0.19 & 0.47 & 0.35 & 0.77 & 0.58 & 0.34 & 0.55 & 0.44 & 0.66 & 0.24 & 0.57 \\
\hline 9 & $-138,400,30$ & 0.98 & 0.25 & 0.55 & 0.38 & 0.15 & 0.04 & 0.13 & 0.19 & 0.19 & 0.6 & 0.09 & 0.56 & 0.14 & 0.69 & 0.07 & 0.6 \\
\hline 10 & $-138,500,30$ & 0.92 & 0.23 & 0.53 & 0.37 & 0.38 & 0.09 & 0.13 & 0.19 & 0.25 & 0.59 & 0.11 & 0.56 & 0.17 & 0.72 & 0.09 & 0.6 \\
\hline
\end{tabular}



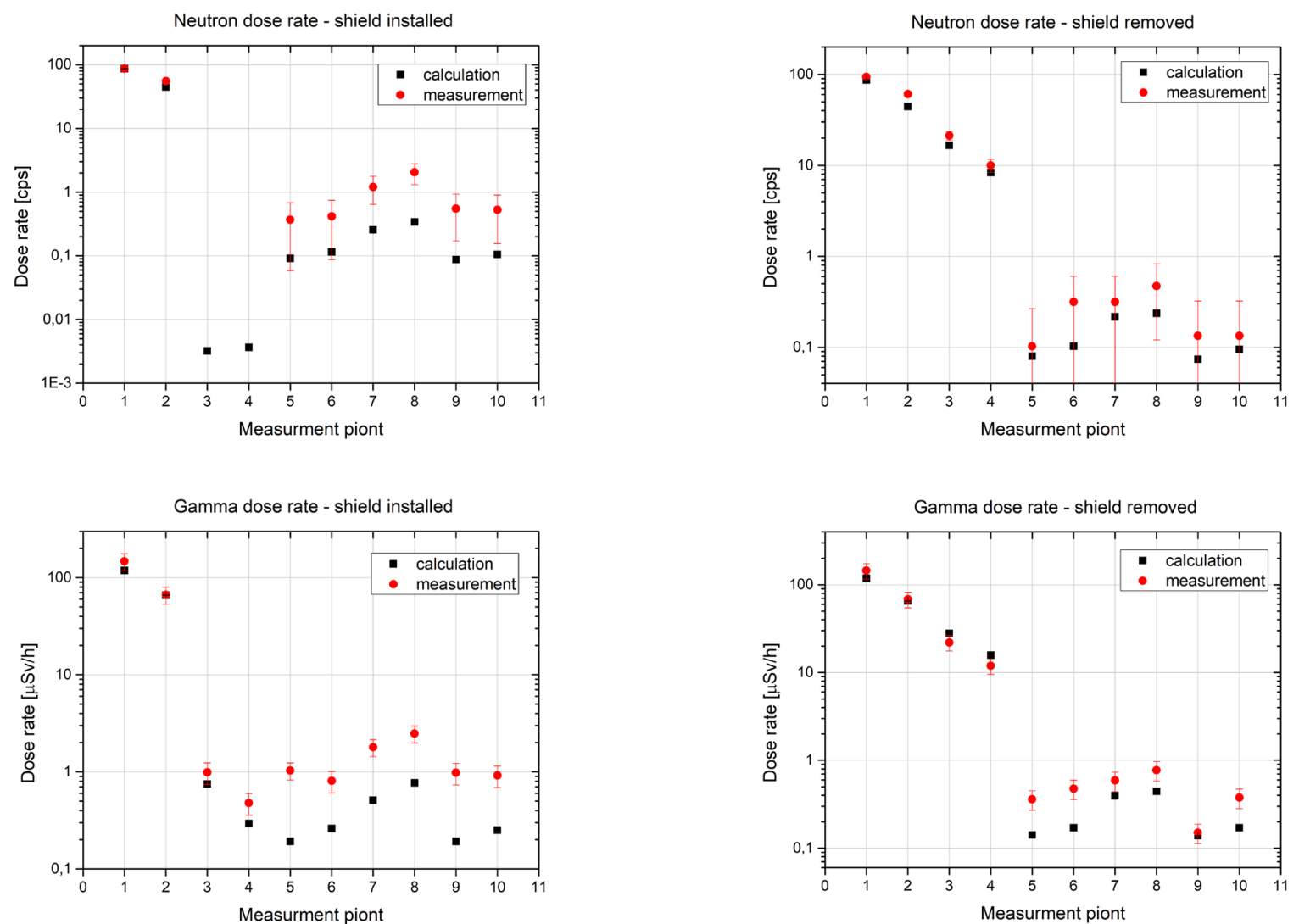

Fig. 5. Comparison of measured and calculated results for neutron dose rate (upper two charts) and gamma dose rate (lower two charts). Left: Concrete and paraffin shield was installed. Right: Shield was removed.

In order to estimate the actual error one would have to take into account all variances in nuclear data, computer model and method applied. However, these approximations are not included in the final variance of the calculated results.

\section{COMPARISON}

Since calculated results were converted to counts per second for neutron dose rate and $\mu \mathrm{Sv} / \mathrm{h}$ for the gamma dose rate, absolute values can be compared. In Table II one can see ratio between calculated result and measured number. For the case where the shield was removed and neutron dose rate was measured (Fig. 5, right side), one can observe good agreement. All calculated results fall within measured error. On the other hand, when the shield in front of the beam tube was present Fig. 5, left side), calculations do not comply with measurements completely. The reason could be in material composition of the paraffin and concrete used for constructing the shield, which is not accurately known and therefore inaccurately modelled. The other reason could be in nuclear data. However, the calculated results follow the trend set by the measurements.

If we compare the measurements and calculations of gamma dose rate, one can see that almost all calculations fall outside error interval of measurements. However, similarly as before, one can see that calculations follow the trend set by the measurements (Fig. 5). It is interesting to compare measurements and calculation for the point 1 (at the beam port entrance). They do not differ for more than $20 \%$, which is good and indicates that most of the deviance is due to the bad model geometry outside the reactor body.

One can see that measurement comply better for the case where shield was removed - cleaner situation. The reason for that can be in not well known material composition of the shield. There could be water inside concrete or some impurities inside paraffin which were not included in the model.

\section{FUTURE WORK AND CONCLUSIONS}

In future, the same set of calculation could be run again, but with different material composition. In that way, it could be possible to estimate the deviation due to unknown material composition of the shield. Furthermore, various parts of the reactor hall will be characterized in the same manner in order to verify the model. When the model will be validated, we will

TABLE II. RATIO BETWEEN CALCULATED AND MEASURED DOSE RATE (GAMMA)S AND CPS (NEUTRON)

\begin{tabular}{|c|c|c|c|c|}
\hline & \multicolumn{2}{|c|}{ Shield installed } & \multicolumn{2}{c|}{ Shield removed } \\
\hline No. & $\gamma$ & Neutron & $\gamma$ & Neutron \\
\hline 1 & 0.80 & 1.00 & 0.81 & 0.93 \\
\hline 2 & 0.99 & 0.81 & 0.96 & 0.73 \\
\hline 3 & 0.76 & $/$ & 1.28 & 0.78 \\
\hline 4 & 0.60 & $/$ & 1.32 & 0.84 \\
\hline 5 & 0.18 & 0.24 & 0.39 & 0.80 \\
\hline 6 & 0.32 & 0.29 & 0.35 & 0.32 \\
\hline
\end{tabular}




\begin{tabular}{|c|c|c|c|c|}
\hline 7 & 0.28 & 0.22 & 0.66 & 0.71 \\
\hline 8 & 0.31 & 0.17 & 0.57 & 0.51 \\
\hline 9 & 0.19 & 0.16 & 0.93 & 0.54 \\
\hline 10 & 0.27 & 0.21 & 0.45 & 0.69 \\
\hline
\end{tabular}

be able to use it also for accidental scenarios, such as loss of water accident. It is important to know expected dose rates during most credible accidents in order to plan sufficient design features of the reactor and apply appropriate emergency procedures to limit received dose of the personnel.

In addition dose rate measurements could serve as benchmark experiments for validation of computer codes for deep penetration problems.

\section{ACKNOWLEDGEMENTS}

I would like to thank Matjaž Stepišnik and Tinkara Bučar from radiation protection unit for helping us with the dose rate measurements around beam port no. 5 .

\section{REFERENCES}

[1] X-5 Monte Carlo Team, MCNP - A General Monte Carlo N-Particle Transport Code, Version 5, Volume I \& II, 2003.

[2] L. Snoj et al., "Computational analysis of irradiation facilities at the JSI TRIGA reactor", Applied Radiation and Isotopes, Vol. 70, pp. 483-488, 2011

[3] K. Ambrožič et al., "Computational analysis of the dose rates at JSI TRIGA reactor irradiation facilities«, Applied Radiation and Isotopes, Vol. 130, pp. 140-152, 2017

[4] L. Snoj et al., "Analysis of neutron flux distribution for the validation of computational methods for the optimization of research reactor utilization", Applied Radiation Isotopes, Vol. 69, pp. 136-141, 2011
[5] S.W. Mosher et. al., ADVANTG - An Automated Variance Reduction Parameter Generator, ORNL/TM-2013/416, Oak Ridge National Laboratory, 2013.

[6] M. B. Chadwick et. al., ENDF/B-VII.0: Next Generation Evaluated Nuclear Data Library for Nuclear Science and Technology, Nuclear Data Sheets, Special Issue on Evaluated Nuclear Data File ENDF/BVII.0, 2006.

[7] T.M. Evans, et. al., Denovo: a new three dimensional parallel discrete ordinates code in SCALE, Nucl. Technol. 171, 171-200, 2010.

[8] J.C. Wagner, 2014. FW-CADIS method for global and semi-global variance reduction of Monte Carlo radiation transport calculations, Nucl. Sci. Eng. 176, 37-57, 2014.

[9] A. Jazbec, L. Snoj, 3D Model of Jožef Stefan Institute TRIGA Mark II Reactor, NENE 2016, Portorož 2016.

[10] ICRP, Data for protection against ionizing from external sources supplement to ICRP Publication 15 ICRP Publication 21. Ann ICRP. 1973.

[11] G. Žerovnik, M. Podvratnik, L. Snoj, On normalization of fluxes and reaction rates in MCNP criticality calculations, Annals of Nuclear Energy, Vol. 63, pp. 126 - 128, 2014

[12] B. Kos, L. Snoj, On using Grasshopper add-on for CAD to MCNP conversion, PHYSOR 2016, Idaho, 2016.

[13] ICRP Publication 103, The 2007 Recommendations of the International Commission on Radiological Protection, 2007.

[14] Operating manual, Neutron Probe LB 6411, Id. No. 1-20188-82042 BA2, Rev. No. 01, 1996. 